\title{
Monitoring of health-related quality of life and symptoms in prostate cancer survivors: a randomized trial
}

Kimberly M. Davis, PhD, ${ }^{1}$ David Dawson, BA, ${ }^{1}$ Scott Kelly, MS, ${ }^{1}$ Sara Red, BA, ${ }^{1}$ Sofiya Penek, BA, ${ }^{1}$ John Lynch, MD, ${ }^{2}$ Sean Collins, MD, ${ }^{3}$ Barlow Lynch, MD, ${ }^{4}$ Michael Porrazzo, MD, ${ }^{4}$ Michael Bass, MA, ${ }^{5}$ and Kathryn L. Taylor, $\mathrm{PhD}^{1}$

${ }^{1}$ Georgetown University Medical Center, Washington, DC; Departments of ${ }^{2}$ Urology and ${ }^{3}$ Radiation Medicine, Georgetown University Medical Center, Washington, DC; ${ }^{4}$ The Washington Hospital Center, Washington, DC; ${ }^{5}$ Northwestern University, Feinberg School of Medicine, Department of Medical Social Science, Chicago, Illinois

Background Routine symptom and health-related quality of life (HRQOL) assessments can engage patients, give provider feedback, and improve doctor/patient communication.

Objective We compared the impact of a technology-assisted symptom monitoring system versus usual care on $\mathrm{HRQOL}$ and doctor/patient communication in early-stage prostate cancer (PCa) survivors.

Methods Men ( $=94$ ) were on average 62-years old, mostly African American (AA; 61.7\%), and 10-19 months post-treatment. They were randomized to symptom monitoring plus feedback $(S M+F ; n=49)$ or usual care $(U C ; n=45)$. SM+F participants completed a 12-item telephone-assisted monitoring intervention. All participants completed a baseline and 2 follow-up interviews.

Results Among the SM+F participants, perceptions of the monitoring system were positive: $97.1 \%$ endorsed it as easy/very easy to use and $85 \%$ felt all patients could benefit from it. At baseline, men reported favorable general and cancer-specific $\mathrm{HRQOL}$ and doctor/patient communication, but poorer urinary and sexual function. Although there was no overall impact of the intervention, post hoc exploratory analyses indicated that among AA men, those who received SM+F improved relative to UC on doctor/patient communication $(P<.05)$, general HRQOL $(P<.06)$, and sexual function $(P<.05)$.

Limitations Variability in survivor follow-up care, limited access to eligible participants, and minimal physician training in the use of reports likely decreased physician investment.

Conclusion Overall, PCa survivors were receptive to this monitoring system. Exploratory analyses suggest that this technologyassisted monitoring system may be of particular benefit to African American men. Additional studies with larger samples, more intervention time-points, and increased physician training are needed to strengthen the intervention's impact.

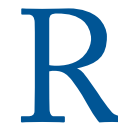

esearch on symptom management and monitoring of health-related quality of life (HRQOL) among cancer patients has typically focused on the active treatment phase. ${ }^{1-7}$ More recently, greater attention has been given to the psychosocial needs and follow-up care plans for survivors. ${ }^{8}$ Several technology-assisted symp-

Manuscript received August 1, 2012; accepted December 12, 2012.

Correspondence Kimberly M. Davis, PhD, Georgetown University Medical Center, Cancer Prevention and Control Program, Department of Oncology, Lombardi Comprehensive Cancer Center, 3300 Whitehaven Street, NW, Suite 4100, Washington, DC 20007 (kmd54@georgetown.edu).

Disclosures The authors completed and submitted the ICMJE Form for Disclosure of Potential Conflicts of Interest and none were reported.

J Support Oncol 2013;11:174-182 @ 2013 Frontline Medical Communications DOI: 10.12788/i.suponc.0013
tom/HRQOL monitoring systems with routine assessments have been shown to be easy to use, ${ }^{1,3,5,9-16}$ readily accepted by patients, ${ }^{3,9,11,14,15,17,18}$ helpful in communication between patients and providers, ${ }^{3,9,11,13,15}$ and a means of overcoming numerous barriers to conducting routine assessments. ${ }^{16,19-23}$ Real-time clinician feedback at the point-of-care appears to be a crucial component of these systems, giving patients and providers a systematic way of discussing symptoms and aspects of HRQOL that are often addressed only informally or not at all.

To date, 6 randomized controlled trials (RCTs) have assessed the impact of technology-assisted interventions among cancer patients. ${ }^{6,23-27}$ There was significant variability across these studies, including differing sample sizes, number of intervention contacts, tumor site (eg, breast, lung, colon), outcomes assessed (eg, symptom distress, com- 
munication, and HRQOL), and types of technology used (eg, touch-screen computers, telephone systems). The methodological differences make it difficult to compare these studies, although a common thread was that patients found the systems easy to use and they generally perceived the systems as beneficial. ${ }^{6,23-27}$

Despite the positive response from participants, only 2 of the $6 \mathrm{RCT}$ s demonstrated positive outcomes for the intervention over the control group. ${ }^{23,25}$ In a study of 286 cancer patients and 28 oncologists, Velikova et al (2004) found that both the intervention and the attentioncontrol groups had better HRQOL than the control group over a 6-month period. ${ }^{23}$ Among the intervention patients, the HRQOL improvement was related to clear use of the HRQOL data by physicians, and to physician/ patient discussion of pain and role function. A positive effect on emotional well-being was associated with feedback of the data to physicians. However, there were no significant differences between the intervention and attention-control groups.

The second RCT with positive findings assessed the symptom severity of 405 oncology clinic patients who reported having depression, pain, or both. ${ }^{25}$ Kroenke et al (2010) demonstrated that centralized telephone-based symptom management coupled with an automated symptom monitoring system resulted in improved pain and depression scores compared to patients who received usual care. The intervention included repeated measurements of symptoms, accompanied by telecare management. Key components for the success of both studies appear to have been active provider participation and extensive provider training.

Across the RCTs that demonstrated negative results, a number of issues were identified, including high attrition rates and use of the same questionnaire for the intervention and the outcome measure, ${ }^{24}$ the need for a longer data collection period, ${ }^{26,28}$ and contamination between the study groups. ${ }^{26}$

Given the call for routine symptom/HRQOL monitoring for cancer survivors, ${ }^{8}$ and the treatment-related side effects that accompany the long survival period following treatment for localized prostate cancer $(\mathrm{PCa})$, we sought to expand this research to $\mathrm{PCa}$ survivors. $\mathrm{PCa}$ is a leading cause of morbidity for American men. ${ }^{29}$ Approximately $90 \%$ of men diagnosed with PCa have early-stage disease and a 5-year survival rate of virtually 100\%. ${ }^{30}$ Most survivors report treatment-related side effects, particularly urinary, bowel, and sexual dysfunction. Although some symptoms resolve within 12 months of treatment completion, long-term treatment-related symptoms are common and have an adverse impact on disease-specific function and HRQOL. ${ }^{31-33}$
To our knowledge, only one study has used a technologyassisted monitoring system to collect HRQOL information from $\mathrm{PCa}$ patients. ${ }^{5}$ Yet, no studies have been conducted with $\mathrm{PCa}$ survivors.

Based on our pilot study ${ }^{34}$ that demonstrated the feasibility of this intervention, we conducted a randomized trial comparing a technology-assisted symptom monitoring system versus usual care on the HRQOL of $\mathrm{PCa}$ survivors. We adapted Kornblith's Vulnerability Model of Psychosocial Adaptation of Cancer Survivors (1998), ${ }^{35}$ an automated symptom/HRQOL monitoring system on prostate cancer survivors' adaptation, to examine the direct and indirect effects of the intervention. We hypothesized that the intervention would improve general and disease-specific HRQOL; management of urinary, bowel, and sexual symptoms; and doctor/patient communication. Additionally, we conducted post hoc exploratory analyses to examine whether the intervention differentially impacted racial subgroups.

\section{Methods}

\section{Participants}

Eligibility criteria included early-stage PCa survivors who were 10-19 months post-treatment; a scheduled follow-up appointment with a urologist or radiation oncologist; the ability to read and understand English; and access to a telephone and the ability to manipulate a telephone keypad to complete the survey.

\section{Procedure}

This study was approved by the Georgetown/Medstar Oncology Institutional Review Board. Participants were recruited from urologists and radiation oncologists at 2 affiliated hospitals in the Washington, DC metropolitan area: Georgetown University Medical Center (GUMC) and Washington Hospital Center (WHC).

Invitation letters were mailed to eligible participants one month prior to their upcoming appointment. One week later, men were called by a research assistant (RA) to further describe the study; obtain verbal informed consent; conduct the baseline (T0) telephone interview; and randomize to either symptom monitoring plus feedback $(\mathrm{SM}+\mathrm{F})$ or usual care (UC). Randomization was conducted using the telephone-based system stored on a server at GUMC. Written consent was obtained via mail following completion of the T0.

Participants completed a total of 3 telephone interviews: baseline (T0), 2 months post-baseline (T1), and approximately 7 months post-baseline (T2). All 3 interviews were conducted by the RA and included the same HRQOL scales. The T2 also included an overall study evaluation. 
- UC Group: UC participants saw their physicians as scheduled but did not use the monitoring system before each follow-up visit and no feedback was provided to physicians.

- SM+F Group: Participants in the SM+F intervention group received written and verbal (by telephone) instructions on how to use the technology-assisted monitoring system.

$\mathrm{SM}+\mathrm{F}$ participants were instructed to call the automated system 3 business days prior to their next 2 follow-up visits with their physician. Reminder calls were made to those who did not call into the system on their own. Men were called every day for up to 3 days, until they either called into the system or had their appointment. For the monitoring intervention, the men completed the Prostate Cancer Subscale (PCS) of the Functional Assessment of Cancer Therapy-Prostate (FACT-P), a 12-item subscale that measures problems specific to prostate cancer. The PCS was used for the intervention so that the intervention itself would not overlap with the outcome measure. Internal consistency ranged from 0.65 to 0.69 . Participants completed the PCS via telephone by responding to questions using their keypad. The responses were stored in a database from which individualized reports were generated. The RA delivered the reports to the physician approximately 24 hours prior to the scheduled follow-up visit. Participants completed a total of 2 monitoring interventions in approximately 7 months.

\section{Description of the monitoring system}

The Prostate Cancer Monitoring System (PCMS) is a telephone/computer-based monitoring system adapted from the Advanced Lung Cancer Management Program (ALCaMP) Study. ${ }^{36}$ ALCaMP was designed to reduce barriers to the routine use of standardized symptom/ HRQOL assessments for patients with advanced lung cancer.

Symptom alerts and report. The report format was based on input from several urologists who participated in the pilot study. ${ }^{34}$ Symptom alerts (the word 'alert' printed beside the patient response) were generated when a patient's endorsement of a symptom was a 4 or 5 on a 5 -point scale, regardless of whether it had changed from the previous assessment. An alert was also generated if the response worsened by 2 or more points from the previous assessment. All items were scored such that higher scores corresponded to more of a particular symptom.

\section{Measures}

Demographic and clinical information. Self-reported $\mathrm{pa}^{-}$ tient information was assessed at T0 and included age, race/ethnicity, education, marital status, employment status, income, treatment, and comorbidities.

General HRQOL. The SF- $12^{37}$ is a 12 -item generic measure of HRQOL composed of 2 subscales, the Mental Component Summary (MCS) and the Physical Component Summary (PCS). It has been widely used among medical patients and the general population. The 2-week test-retest reliability for the MCS was 0.76 and 0.89 for the PCS. ${ }^{37,38}$ Higher scores indicate better general HRQOL. ${ }^{38}$ The SF-12 was administered at T0, T1, and $\mathrm{T} 2$.

Cancer-specific HRQOL. The Functional Assessment of Cancer Therapy - General (FACT-G) ${ }^{39}$ is a 27-item questionnaire divided into $4 \mathrm{HRQOL}$ domains: Physical Well-Being (PWB), Social Well-Being (SWB), Emotional Well-Being (EWB), and Functional WellBeing (FWB). The FACT-G is a well-validated and widely used cancer-specific questionnaire. The total score ranges from 0 to 108 , with a higher score indicating better quality of life. The FACT-G was administered at T0, TI and $\mathrm{T} 2$.

Prostate cancer-specific HRQOL. The UCLA Prostate Cancer Index (UCLA-PCI) ${ }^{40}$ is a 20 -item measure of function and bother in urinary, bowel, and sexual domains for men treated for PCa. Higher scores indicate better functioning. The UCLA PCI was also administered at $\mathrm{T} 0$, $\mathrm{T} 1$, and $\mathrm{T} 2$.

Doctor/patient communication. The Primary Care Assessment Survey (PCAS) is a measure originally designed for the primary care setting, although it has also been used in the oncology setting. ${ }^{41}$ We included 2 of the 11 subscales: communication (6 items) and interpersonal treatment (5 items). The 6-point response scale included categories from 'Very poor' to 'Excellent'. Scores ranged from 0-100 points, with higher scores indicating more favorable ratings. ${ }^{42}$ Overall satisfaction with care by the doctor was measured by one item with responses ranging from poor "0" to excellent " 10 ". The PCAS was also administered at T0, T1, and T2.

Post-visit ratings (PVR). Patients and physicians rated their perceptions about how well symptoms/ HRQOL issues were addressed. Patients and physicians rated activities that were performed during the clinic visit, including referrals to other healthcare providers (eg, psychiatry, physical therapy) and prescriptions and/or other activities performed in response to patient-reported concerns. PVRs were administered after each physician visit. Patient data for both groups was above $80 \%$ following each visit while physician data was approximately $60 \%$ for patients in both groups. These discrepancies made it difficult to make comparisons between patients and physicians and thus these data are not discussed further. 
Patient/physician study evaluation. At the T2 assessment, $\mathrm{SM}+\mathrm{F}$ participants completed a 10-item questionnaire to evaluate the utility and acceptability of the monitoring system and their study participation. UC participants completed a 7-item questionnaire to evaluate their study participation. At the end of the study, physicians completed a 10-item questionnaire to evaluate the impact of study participation on their communication with patients and their satisfaction with the care they provided.

Data analysis. Intent-to-treat analyses included all patients who were randomized in the study $(\mathrm{N}=94)$. We present comparisons on the outcomes at T2 (ie, following the opportunity for completion of both interventions). We conducted descriptive statistics, $t$-tests, and chi-square statistics to determine whether there were any baseline group differences on demographic, clinical, or outcome variables. Multivariate analyses included repeated measures ANCOVAs to compare $\mathrm{SM}+\mathrm{F}$ and $\mathrm{UC}$ participants on the primary outcomes. We also conducted per-protocol analyses, limited to the $83 \%$ in the $\mathrm{SM}+\mathrm{F}$ group who had completed both monitoring interventions. Finally, we conducted post hoc exploratory repeated measures ANCOVAs with race as a factor to examine whether the intervention had a differential impact within racial subgroups. All multivariate analyses were adjusted for age and education due to significant univariate associations between these variables and the outcome variables. We used the Statistical Package for the Social Sciences (SPSS) versions 19 and 20 to conduct the analyses.

\section{Results}

\section{Participation and retention rates}

We contacted 142 men with early-stage PCa (Figure 1). Ten men were ineligible (eg, non-English speakers, disconnected phone numbers, cognitive impairment). Of the 132 eligible men, 94 (71\%) agreed to participate in the study (SM+F group $[\mathrm{n}=49]$ vs UC group $[\mathrm{n}=45])$. Retention rates are presented in Figure 1. T2 retention rates are based on the number of eligible participants at T0 who had a final follow-up appointment. Reasons for study dropout at T1 and T2 included: not comfortable talking about $\mathrm{PCa}$, unreachable, too busy, confidentiality issues, too ill, and moved out of the country.

Demographic characteristics are shown in Table 1. Participants were a mean age of 62-years, and over onehalf were African American (AA; 61.7\%), married (78.7\%), had a college/advanced degree (50.0\%), were working full-time (60.6\%), and had an annual household income over $\$ 100,000$ (57.4\%). Approximately 44\% had 2 or more comorbid illnesses and the majority had had a radical prostatectomy (RP). There were no significant differences between the groups on any demographic or clinical variables at $\mathrm{T} 0$.

\section{Participants' evaluation of the study}

At the T2 interview, all participants completed an evaluation of their participation experience. Among the $\mathrm{SM}+\mathrm{F}$ participants, the majority (97.1\%) endorsed that it was easy or very easy to use the monitoring system and $85 \%$ endorsed that all patients would benefit from an automated monitoring system to routinely assess their symptoms/HRQOL. When asked how often they would like to complete a symptom assessment, $62 \%$ reported before each visit, $14 \%$ every other month, 5\% monthly, and 19\% never. Finally, $65 \%$ of SM+F participants said an automated symptom monitoring system would be 'quite a bit' or 'very useful' for general patient care following treatment.

Approximately $88 \%$ of all participants indicated that the interview questions asked were important, $84 \%$ reported that the questions were not too personal, and $83 \%$ reported that the surveys were not too long to complete. When asked about their preference for mode of completing a survey, $66 \%$ endorsed the computer/internet while $16 \%$ endorsed the telephone, $13 \%$ endorsed no preference and $6 \%$ preferred in-person surveys.

Process variables. At T1, 92\% of the SM+F participants completed the intervention, and at T2 $85 \%$ completed the intervention. Overall, $96 \%$ were exposed to at least one and $83 \%$ were exposed to both interventions.

Comparison of $\mathrm{SM}+\mathrm{F}$ and $\mathrm{UC}$ groups on study outcomes. At baseline, patients reported very favorable levels of general HRQOL (SF-12), cancer-specific HRQOL (FACT-G), bowel functioning (UCLA-PCI), and doctor/patient communication (PCAS). Only the sexual and urinary functioning scores suggested a low level of disease-specific functioning (ULCA-PCI). None of the measures differed significantly between groups at baseline (Table 2). We conducted intent-to-treat repeated measures ANCOVA analyses to assess the impact of the intervention. Contrary to our hypotheses, the multivariate analyses revealed no significant group by time interactions on any of the outcomes at T2 (all P's $>$.10). We also found no significant group differences when we limited the analyses to those who were exposed to the intervention.

Moderator analyses. To assess whether the intervention had a differential impact within racial subgroups (AA/White), we conducted post hoc exploratory repeated measures ANCOVAs that included race as a factor with education and age as covariates. On the sexual function subscale, there was a group by time by race interaction $(\mathrm{F}(1,59)=4.15 ; P=.05)$, revealing that $\mathrm{AA}$ men in the $\mathrm{SM}+\mathrm{F}$ group improved more than White men in the $\mathrm{SM}+\mathrm{F}$ group and also more than AA men in the UC arm (Figure 2). On the overall subscale of the PCAS, there was a significant group by time by race interaction $(\mathrm{F}(1,61)=6.28 ; P=.02)$, 


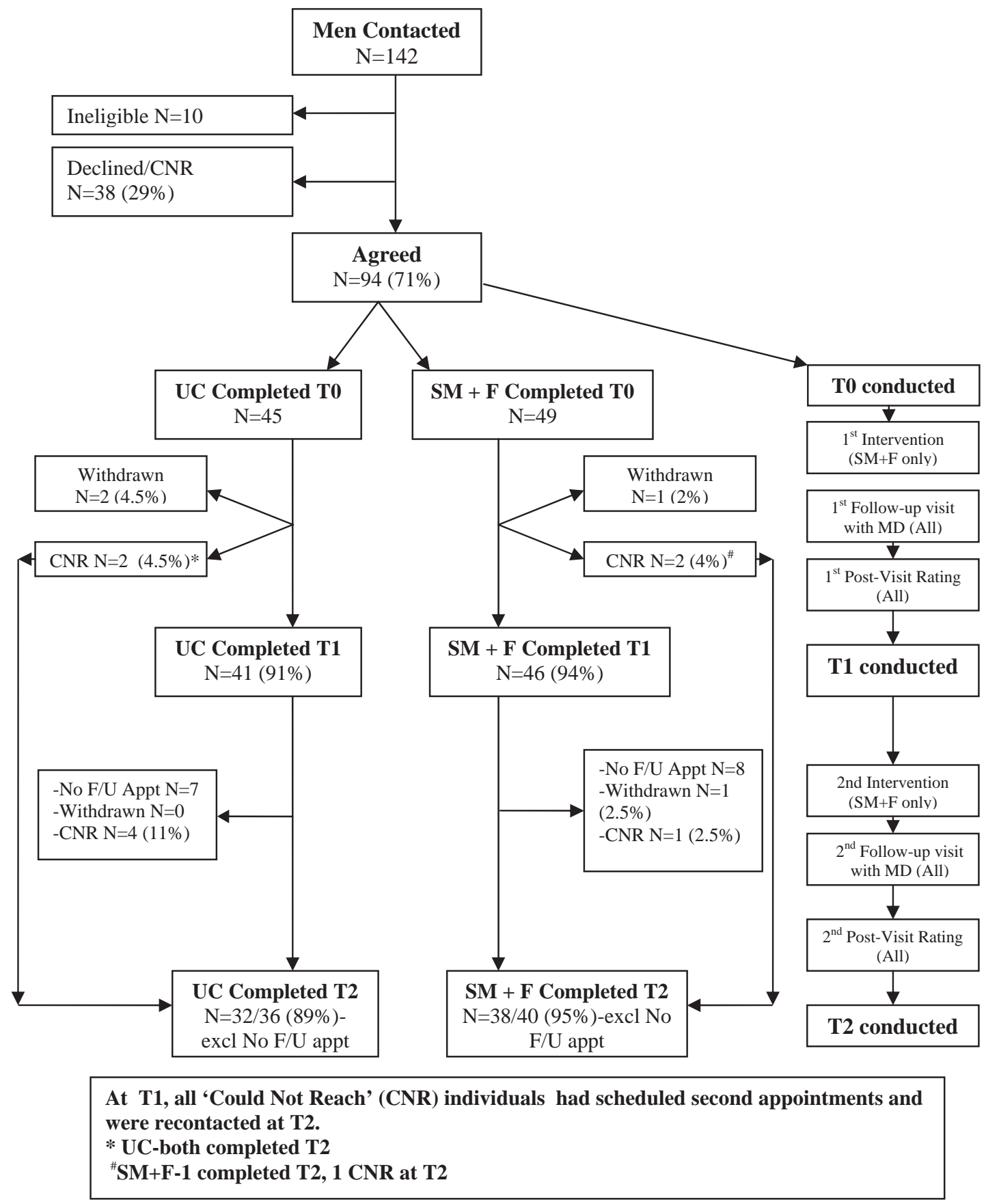

FIGURE 1 PCMS study flowchart.

indicating that White men in the UC group improved while those in the $\mathrm{SM}+\mathrm{F}$ group declined. The reverse was true for $\mathrm{AA}$ men: those in the $\mathrm{SM}+\mathrm{F}$ group improved while those in the UC group declined (Figure 3). Finally, for the MCS analysis, there was a marginally significant group by time by race interaction $(\mathrm{F}(1,61)=$ 3.73; $P=.06)$, which revealed that White men in both the $\mathrm{UC}$ and $\mathrm{SM}+\mathrm{F}$ groups declined, while $\mathrm{AA}$ men in the UC group declined but those in the $\mathrm{SM}+\mathrm{F}$ group improved (Figure 4). Across all 3 figures, AA men in the $\mathrm{SM}+\mathrm{F}$ intervention improved compared to $\mathrm{AA}$ men in the UC group. Among White men, the SM+F intervention resulted in an improvement over the UC group only on the sexual function outcome. The interactions with 
TABLE 1 Sample characteristics/clinical information

\begin{tabular}{|c|c|c|c|}
\hline Sample characteristics & $S M+F(N=49)$ & UC $(N=45)$ & Total $(N=94)$ \\
\hline Age (years) (Mean, SD) & $61.9(7.0)$ & $62.0(8.1)$ & $62.0(7.5)$ \\
\hline Missing & 1 & & 1 \\
\hline $48-60$ years & $24(50.0)$ & $21(46.7)$ & $45(48.4)$ \\
\hline $61-78$ years & 24 (50.0) & $24(53.3)$ & $48(51.6)$ \\
\hline Missing & 1 & & 1 \\
\hline White & $16(32.7)$ & $17(37.8)$ & $33(35.1)$ \\
\hline African American & $31(63.3)$ & $27(60.0)$ & $58(61.7)$ \\
\hline Other & $1(2.0)$ & $1(2.2)$ & $2(2.1)$ \\
\hline Refused & $1(2.0)$ & 0 & $1(1.1)$ \\
\hline \multicolumn{4}{|l|}{ Marital status N (\%) } \\
\hline High school or less & $24(49.0)$ & $23(51.1)$ & $47(50.0)$ \\
\hline College/advanced degree & $25(51.0)$ & 22 (48.9) & $47(50.0)$ \\
\hline \multicolumn{4}{|l|}{ Household income N (\%) } \\
\hline$\leq \$ 100,000$ & 18 (36.7) & $21(46.7)$ & $39(41.5)$ \\
\hline$\geq \$ 100,000$ & $31(63.3)$ & $23(51.1)$ & $54(57.4)$ \\
\hline Missing & & 1 & 1 \\
\hline \multicolumn{4}{|l|}{ Employment status $\mathrm{N}(\%)$} \\
\hline Full time & $31(63.3)$ & $26(57.8)$ & $57(60.6)$ \\
\hline Retired/parttime/else & $18(36.7)$ & $19(42.2)$ & 37 (39.4) \\
\hline RT only (Inc. 1 RP + RT) & $7(14.3)$ & $9(20.0)$ & $16(17.0)$ \\
\hline ADT + other & $9(18.4)$ & $7(15.6)$ & $16(17.0)$ \\
\hline Watchful waiting & $2(4.1)$ & $2(4.4)$ & $4(4.3)$ \\
\hline \multicolumn{4}{|l|}{ Site $N(\%)$} \\
\hline GUMC & 27 (52.9) & $24(47.1)$ & $51(54.3)$ \\
\hline WHC & $22(51.2)$ & $21(48.9)$ & $43(45.7)$ \\
\hline
\end{tabular}

Abbreviations: ADT, androgen deprivation therapy; GUMC, Georgetown Univeristy Medical Center; RP, radical prostatectomy; RT, radiation therapy; SM+F, symptom monitoring plus feedback; UC, usual care; WHC, Washington Hospital Center.

No statistically significant differences were found between the 2 groups.

race were nonsignificant for the remaining outcome $>$ measures (FACT-G total score, all FACT-G subscale scores, the PCS of the SF-12, urinary function and bowel function of the UCLA PCI, and the Communication and Interpersonal subscales of the PCAS).

\section{Discussion}

The objective of this RCT was to compare the impact of a technology-assisted monitoring system versus usual care on HRQOL and doctor/patient communication in $\mathrm{PCa}$ survivors. Our hypotheses regarding overall group differ- 
TABLE 2 Primary outcome measure means by randomization group across time

\begin{tabular}{|c|c|c|c|c|}
\hline \multirow{2}{*}{$\begin{array}{c}\text { Timepoint } \rightarrow \\
\text { Group } \rightarrow \\
\text { Outcomes } \downarrow\end{array}$} & \multicolumn{2}{|c|}{ TO } & \multicolumn{2}{|c|}{ T2 } \\
\hline & $S M+F(38)$ & UC (32) & $S M+F(38)$ & UC (32) \\
\hline FACT-G & $M(S D)$ & $M(S D)$ & $M(S D)$ & $M(S D)$ \\
\hline Total score & $93.3(7.6)$ & $94.2(12.1)$ & 92.5 (12.3) & 94.8 (11.3) \\
\hline Physical well-being & $26.0(2.1)$ & $25.6(2.3)$ & $25.5(3.6)$ & $25.9(2.6)$ \\
\hline Social well-being & $21.6(3.7)$ & $22.4(4.6)$ & $21.8(4.4)$ & $23.1(3.2)$ \\
\hline Emotional well-being & $21.7(2.3)$ & $21.7(3.0)$ & $21.6(2.5)$ & $21.7(3.1)$ \\
\hline Functional well-being & $24.0(3.2)$ & $24.6(4.3)$ & $23.6(4.6)$ & $24.1(4.4)$ \\
\hline \multicolumn{5}{|l|}{ SF-12 } \\
\hline Mental component subscale & $56.5(4.0)$ & $56.0(5.4)$ & $55.1(7.8)$ & $53.8(7.8)$ \\
\hline Physical component subscale & $51.1(6.5)$ & $50.7(7.3)$ & $50.5(8.1)$ & $53.8(5.1)$ \\
\hline \multicolumn{5}{|l|}{ UCLA-PCI } \\
\hline Urinary function & $59.2(13.7)$ & $60.8(12.3)$ & $60.2(14.4)$ & 60.8 (13.7) \\
\hline Bowel function & $89.4(13.2)$ & $91.9(8.6)$ & $88.5(14.3)$ & 90.5 (14.7) \\
\hline Sexual function & $33.9(25.7)$ & $39.9(26.1)$ & $45.3(29.6)$ & $45.5(30.6)$ \\
\hline Missing & 1 & 1 & 1 & 1 \\
\hline \multicolumn{5}{|l|}{ PCAS } \\
\hline Communication & 85.4 (15.9) & $83.6(17.3)$ & 86.7 (12.9) & 84.8 (16.5) \\
\hline Interpersonal & $82.4(17.6)$ & $85.0(15.2)$ & $82.9(16.8)$ & 84.1 (18.0) \\
\hline Overall & $82.6(10.6)$ & 82.5 (12.2) & $83.7(8.8)$ & $84.4(9.5)$ \\
\hline
\end{tabular}

Abbreviations: FACT-G, Functional Assessment of Cancer Therapy-General; PCAS, Primary Care Assessment Survey; SM+F, symptom monitoring plus feedback; UC, usual care; UCLA-PCI, UCLA Prostate Cancer Index.

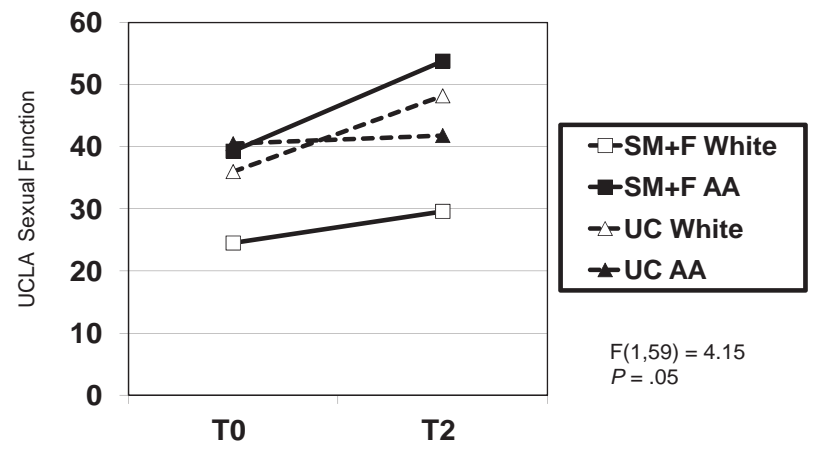

FIGURE 2 Exploratory analyses regarding intervention's impact within racial subgroups. UCLA sexual function. Abbreviations: $\mathrm{SM}+\mathrm{F}$ White, symptom monitoring + feedback White; $\mathrm{SM}+\mathrm{FAA}$ symptom monitoring + feedback African American; UC White, usual care White; UC AA, usual care African American.

ences were unconfirmed, potentially due to the very high baseline scores on the majority of our outcome measures, which made it difficult to demonstrate change over time. However, post hoc exploratory analyses suggested that the intervention benefited $\mathrm{AA}$ men in the $\mathrm{SM}+\mathrm{F}$ group compared to AA men in the $\mathrm{UC}$ group while the $\mathrm{SM}+\mathrm{F}$ intervention did not have the same beneficial impact among White men. Although these findings will need

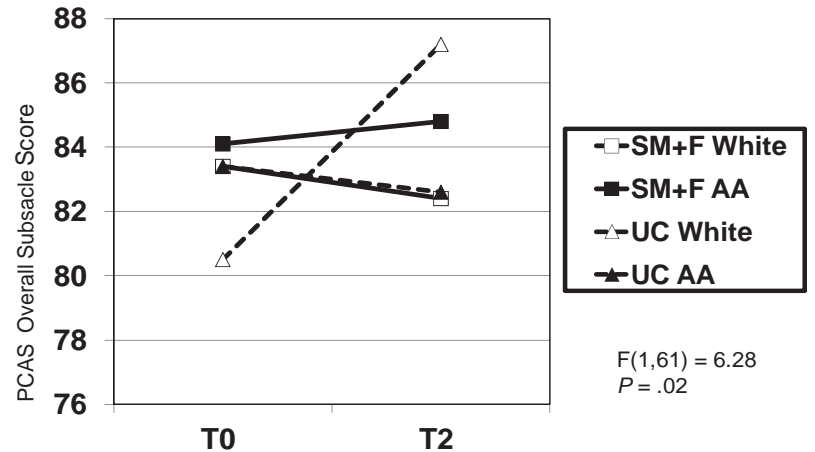

FIGURE 3 Exploratory analyses regarding intervention's impact within racial subgroups. PCAS overall subscale. Abbreviations: $\mathrm{SM}+\mathrm{F}$ White, symptom monitoring + feedback White; $\mathrm{SM}+\mathrm{FAA}$, symptom monitoring + feedback African American; UC White, usual care White; UC AA, usual care African American.

replication, this technology-assisted monitoring system may have provided AA men with a tool that highlights their symptom/HRQOL issues and helps them communicate with their physicians.

Despite the fact that this was a negative study with respect to HRQOL outcomes, this study makes several important contributions to the literature on symptom/ 


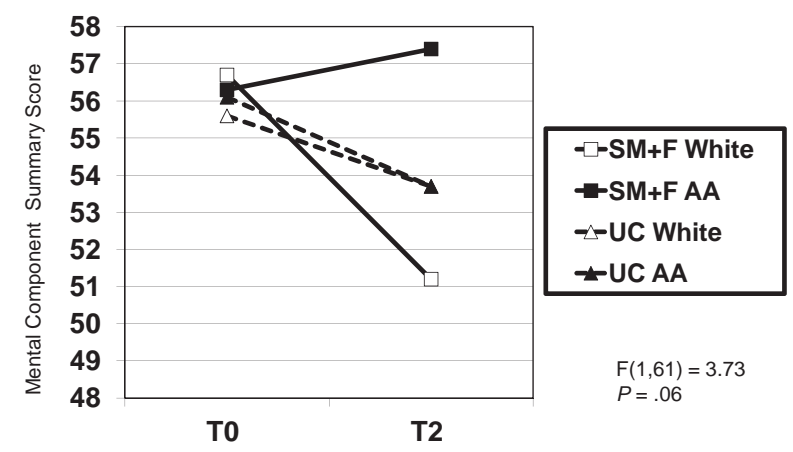

Note. These are adjusted means (adjusting for age, education and the baseline measure of the outcome).

FIGURE 4 Exploratory analyses regarding intervention's impact within racial subgroups. MCS 12. Abbreviations: $S M+F$ White, symptom monitoring + feedback White; $S M+F A A$, symptom monitoring + feedback African American; UC White, usual care White; UC AA, usual care African American.

HRQOL monitoring, particularly about the process issues. First, this study is, to our knowledge, the first RCT examining the impact of a monitoring system focused on symptoms/HRQOL in cancer survivors, with a focus on $\mathrm{PCa}$ survivors. This research addressed survivorship issues, specifically in $\mathrm{PCa}$ survivors who are known to experience significant side-effects following treatment. We expected that this system would be particularly useful for men as they tend to report fewer symptoms/problems compared to women ${ }^{43-45}$ and may be more likely to express their concerns in a less direct manner. Because this system was successfully adapted from advanced lung cancer patients to $\mathrm{PCa}$ survivors, it may be used with patients along the trajectory of cancer care to evaluate HRQOL and other important outcomes such as doctor/patient communication. Finally, our results suggest that this type of system may be of particular benefit to AA men. However, additional studies are needed to confirm these findings.

Study limitations included the significant variability in follow-up care for $\mathrm{PCa}$ survivors. For example, men who received RP tended to see their urologist only every 6 months while men who had combination therapy may have alternated between their radiation oncologist and their urologist every 3 months. This difference in follow-up visits may have posed an unanticipated problem for recruitment of eligible participants for this study. Second, because eligibility was tied to a follow-up visit for men approximately one year post-completion of treatment, many men may have already stopped regular follow-up with these specialists by that time. Primary care physicians may provide an additional avenue for recruitment of $\mathrm{PCa}$ survivors.

An important design consideration was the concern regarding contamination between groups because physicians treated patients in both groups. In subsequent studies, randomization by site will be necessary to address this concern. In the present study, we met with each physician prior to the start of the study to provide a study overview and to show them a sample report that they would be given prior to each $\mathrm{SM}+\mathrm{F}$ participant clinic visit. In retrospect, we recognize that increased physician training in the use of these types of reports may have engendered greater investment from physicians as evidenced by Velikova. ${ }^{23}$ Future studies must train and incentivize physicians to complete study ratings so that comparisons between patient and physician data can be analyzed. Physicians must also understand the importance of completing the study evaluations so that they can share what worked and what did not regarding the information gained about problems experienced by their patients. A final study design consideration was the number of interventions. In the present study, participants completed 2 interventions. Other studies had more than 2 interventions, which have been identified by researchers as a potential factor that may impact the results. ${ }^{27}$ If a study such as this were started just after the completion of treatment, having additional interventions may result in a greater impact over time.

\section{Future directions}

Additional research is needed to continue to assess the use of technology-assisted systems for the routine symptom and HRQOL assessment for cancer patients and survivors. Needed improvements to this type of research include larger sample sizes, more intervention points, more physician training, and randomization by site. Finally, further efforts to explore the impact of such interventions on doctor/patient communication need to be examined as little research in this area has been conducted. Automated monitoring for cancer patients and survivors is an important area of research that could have significant clinical impact by allowing patients and survivors to have a more active role in their follow-up care. Determining cost-effective methods for using these types of systems is also needed to improve the long-term outcomes of cancer survivors.

\section{Acknowledgements}

The authors are grateful to all the participants and physicians who participated in the study.

Funding/support. This work was supported by a grant from the National Cancer Institute R03 - CA119765-01A1a.

Role of sponsor. The National Cancer Institute had no role in the design and conduct of the study or the collection, management, analysis, and interpretation of the data.

\section{References}

1. Erharter A, Giesinger J, Kemmler G, et al. Implementation of computer-based quality-of-life monitoring in brain tumor outpatients in routine clinical practice. J Pain Symptom Manage. 2010;39(2):219-229.

2. Fortner B, Okon T, Schwartzberg L, Tauer K, Houts AC. The Cancer Care Monitor: psychometric content evaluation and pilot testing of a computer administered system for symptom screening and quality of life in adult cancer patients. J Pain Symptom Manage. 2003;26(6):1077-1092. 
3. Head BA, Studts JL, Bumpous JM, et al. Development of a telehealth intervention for head and neck cancer patients. Telemed J E Health. 2009;15(1):44-52.

4. Krouse RS, Herrinton LJ, Grant M, et al. Health-related quality of life among long-term rectal cancer survivors with an ostomy: manifestations by sex. J Clin Oncol. 2009;27(28):4664-4670.

5. Matthew AG, Currie KL, Irvine J, et al. Serial personal digital assistant data capture of health-related quality of life: a randomized controlled trial in a prostate cancer clinic. Health Qual Life Outcomes. 2007;5:38.

6. Sikorskii A, Given CW, Given B, et al. Symptom management for cancer patients: a trial comparing two multimodal interventions. J Pain Symptom Manage. 2007;34(3):253-264.

7. So WK, Marsh G, Ling WM, et al. The symptom cluster of fatigue, pain, anxiety, and depression and the effect on the quality of life of women receiving treatment for breast cancer: a multicenter study. Oncol Nurs Forum. 2009;36(4):E205-E214.

8. National Cancer Institute, National Institutes of Health, U.S. Department of Health and Human Services. Living beyond cancer: finding a new balance. President's Cancer Panel 2003-2004 Annual Report. http://deainfo.nci.nih.gov/advisory/pcp/annualReports/pcp03-04rpt/ Survivorship.pdf. Accessed April 30, 2012.

9. Abernethy AP, Herndon JE, Wheeler JL, et al. Feasibility and acceptability to patients of a longitudinal system for evaluating cancer-related symptoms and quality of life: pilot study of an e/Tablet data-collection system in academic oncology. J Pain Symptom Manage. 2009;37(6): 1027-1038.

10. Chang CH, Cella D, Masters GA, et al. Real-time clinical application of quality-of-life assessment in advanced lung cancer. Clin Lung Cancer. 2002;4(2):104-109.

11. Davis K, Dawson D, Kelly S, et al. Using a routine symptom/healthrelated quality of life monitoring system in prostate cancer survivors. Presented at the 5th Biennial Cancer Survivorship Research Conference, Washington, DC, June 2010.

12. Huang HY, Wilkie DJ, Zong SP, et al. Developing a computerized data collection and decision support system for cancer pain management. Comput Inform Nurs. 2003;21(4):206-217.

13. Kearney N, Kidd L, Miller M, et al. Utilising handheld computers to monitor and support patients receiving chemotherapy: results of a UKbased feasibility study. Support Care Cancer. 2006;14(7):742-752.

14. Mullen KH, Berry DL, Zierler BK. Computerized symptom and quality-of-life assessment for patients with cancer part II: acceptability and usability. Oncol Nurs Forum. 2004;31(5):E84-E89.

15. Wilkie DJ, Huang HY, Berry DL, et al. Cancer symptom control: feasibility of a tailored, interactive computerized program for patients. Fam Community Health. 2001;24(3):48-62.

16. Wright EP, Selby PJ, Crawford M, et al. Feasibility and compliance of automated measurement of quality of life in oncology practice. J Clin Oncol. 2003;21(2):374-382.

17. Berry DL, Trigg LJ, Lober WB, et al. Computerized symptom and quality-of-life assessment for patients with cancer part I: development and pilot testing. Oncol Nurs Forum. 2004;31(5):E75-E83.

18. Carpenter JS, Rawl S, Porter J, et al. Oncology outpatient and provider responses to a computerized symptom assessment system. Oncol Nurs Forum. 2008;35(4):661-669.

19. Detmar SB, Muller MJ, Schornagel JH, Wever LD, Aaronson NK. Health-related quality-of-life assessments and patient-physician communication: a randomized controlled trial. JAMA. 2002;288(23):30273034.

20. Espallargues M, Valderas JM, Alonso J. Provision of feedback on perceived health status to health care professionals: a systematic review of its impact. Med Care. 2000;38(2):175-186.

21. Committee on Quality Health Care in America, Institute of Medicine. Crossing the quality chasm: a new health system for the 21st century. Washington, DC: National Academy Press; 2001.

22. Naughton M, Homsi J. Symptom assessment in cancer patients. Cur Oncol Rep. 2002;4(3):256-263.

23. Velikova G, Booth L, Smith AB, et al. Measuring quality of life in routine oncology practice improves communication and patient wellbeing: a randomized controlled trial. J Clin Oncol. 2004;22(4):714-724.
24. Kearney N, McCann L, Norrie J, et al. Evaluation of a mobile phonebased, advanced symptom management system (ASyMS) in the management of chemotherapy-related toxicity. Support Care Cancer. 2009; 17(4):437-444.

25. Kroenke $\mathrm{K}$, Theobald D, Wu J, et al. Effect of telecare management on pain and depression in patients with cancer: a randomized trial. JAMA. 2010;304(2):163-171.

26. McLachlan SA, Allenby A, Matthews J, et al. Randomized trial of coordinated psychosocial interventions based on patient self-assessments versus standard care to improve the psychosocial functioning of patients with cancer. I Clin Oncol. 2001;19(21):4117-4125.

27. Taenzer P, Bultz BD, Carlson LE, et al. Impact of computerized quality of life screening on physician behaviour and patient satisfaction in lung cancer outpatients. Psychooncology. 2000;9(3):203-213.

28. McCann L, Maguire R, Miller M, Kearney N. Patients' perceptions and experiences of using a mobile phone-based advanced symptom management system (ASyMS) to monitor and manage chemotherapy related toxicity. Eur J Cancer Care (Engl). 2009;18(2):156-164.

29. Siegel R, Naishadham D, Jemal A. Cancer statistics, 2012. CA Cancer J Clin. 2012;62(1):10-29.

30. Jemal A, Siegel R, Xu J, Ward E. Cancer statistics, 2010. CA Cancer J Clin. 2010;60(5):277-300.

31. Cooperberg MR, Broering JM, Litwin MS, et al. The contemporary management of prostate cancer in the United States: lessons from the cancer of the prostate strategic urologic research endeavor (CapSURE), a national disease registry. J Urol. 2004;171(4):1393-1401.

32. Potosky AL, Davis WW, Hoffman RM, et al. Five-year outcomes after prostatectomy or radiotherapy for prostate cancer: the prostate cancer outcomes study. J Natl Cancer Inst. 2004;96(18):1358-1367.

33. Taylor KL, Luta G, Miller AB, et al. Long-term disease-specific functioning among prostate cancer survivors and noncancer controls in the prostate, lung, colorectal, and ovarian cancer screening trial. J Clin Oncol. 2012;30(22):2768-2775.

34. Davis K, Dawson D, Lynch J, et al. Routine symptom/health-related quality of life monitoring in prostate cancer survivors: a pilot study. [Abstract \#2158]. Presented at the Society of Behavioral Medicine 28th Annual Meeting and Scientific Sessions, Washington, DC, March 2007.

35. Kornblith AB. Psychosocial adaptation of cancer survivors. In: Holland JC, Breitbart W, Jacobsen PB, et al, editors. Psycho-oncology. New York: Oxford University Press; 1998. p. 223-41.

36. Davis K, Yount S, Del Ciello K, et al. An innovative symptom monitoring tool for people with advanced lung cancer: a pilot demonstration. J Support Oncol. 2007;5(8):381-387.

37. Ware J, Jr., Kosinski M, Keller SD. A 12-Item Short-Form Health Survey: construction of scales and preliminary tests of reliability and validity. Med Care. 1996;34(3):220-233.

38. Ware JE, Kosinski M, Keller SD. SF-12: How to score the SF-12 Physical and Mental Health Summary Scales. 3rd ed. Lincoln, RI: QualityMetric Inc.; 1998.

39. Cella DF, Tulsky DS, Gray G, et al. The Functional Assessment of Cancer Therapy scale: development and validation of the general measure. J Clin Oncol. 1993;11(3):570-579.

40. Litwin MS, Hays RD, Fink A, Ganz PA, Leake B, Brook RH. The UCLA Prostate Cancer Index: development, reliability, and validity of a health-related quality of life measure. Med Care. 1998;36(7):1002-1012.

41. Fleming DA, Sheppard VB, Mangan PA, et al. Caregiving at the end-of-life: perceptions of health care quality and quality of life among patients and caregivers. J Pain Symptom Manage. 2006;31(5):407-420.

42. Safran DG, Kosinski M, Tarlov AR, et al. The Primary Care Assessment Survey: tests of data quality and measurement performance. Med Care. 1998;36(5):728-739.

43. Barsky AJ, Peekna HM, Borus JF. Somatic symptom reporting in women and men. J Gen Intern Med. 2001;16(4):266-275.

44. Galovski TE, Mott J, Young-Xu Y, Resick PA. Gender differences in the clinical presentation of PTSD and its concomitants in survivors of interpersonal assault. J Interpers Violence. 2011;26(4):789-806.

45. Watson L, Vestbo J, Postma DS, et al. Gender differences in the management and experience of Chronic Obstructive Pulmonary Disease. Respir Med. 2004;98(12):1207-1213. 